\title{
Use of rumination and activity monitoring for the identification of dairy cows with health disorders: Part III. Metritis
}

\author{
M. L. Stangaferro, R. Wijma, L. S. Caixeta, ${ }^{1}$ M. A. Al-Abri, ${ }^{2}$ and J. O. Giordano ${ }^{3}$ \\ Department of Animal Science, Cornell University, Ithaca, NY 14853
}

\begin{abstract}
The objectives of this study were to evaluate (1) the performance of an automated health-monitoring system (AHMS) to identify cows with metritis based on an alert system (health index score, HIS) that combines rumination time and physical activity; (2) the number of days between the first HIS alert and clinical diagnosis (CD) of metritis by farm personnel; and (3) the daily rumination time, physical activity, and HIS patterns around $\mathrm{CD}$. In this manuscript, the overall performance of HIS to detect cows with all disorders of interest in this study [ketosis, displaced abomasum, indigestion (companion paper, part I), mastitis (companion paper, part II), and metritis] is also reported. Holstein cattle ( $\mathrm{n}=1,121 ; 451$ nulliparous and 670 multiparous) were fitted with a neck-mounted electronic rumination and activity monitoring tag (HR Tags, SCR Dairy, Netanya, Israel) from at least -21 to $80 \mathrm{~d}$ in milk (DIM). Raw data collected in 2 -h periods were summarized per $24 \mathrm{~h}$ as daily rumination and activity. An HIS (0 to 100 arbitrary units) was calculated daily for individual cows with an algorithm that used rumination and activity. A positive HIS outcome was defined as an HIS of $<86$ units during at least $1 \mathrm{~d}$ from -5 to $2 \mathrm{~d}$ after CD. Blood concentrations of nonesterified fatty acids, $\beta$-hydroxybutyrate, total calcium, and haptoglobin were determined in a subgroup of cows $(\mathrm{n}=459)$ at $-11 \pm 3,-4 \pm 3,0,3 \pm 1,7 \pm 1,14 \pm 1$, and $28 \pm$ 1 DIM. The overall sensitivity of HIS was $55 \%$ for all cases of metritis $(\mathrm{n}=349)$, but it was greater for cows with metritis and another disorder (78\%) than for cows with metritis only (53\%). Cows diagnosed with metritis and flagged based on HIS had substantial alterations in
\end{abstract}

\footnotetext{
Received April 22, 2016.

Accepted May 21, 2016.

${ }^{1}$ Current address: Department of Clinical Sciences, College of Veterinary Medicine and Biomedical Sciences, Colorado State University, Fort Collins, CO, 80523.

${ }^{2}$ Current address: Department of Animal and Veterinary Sciences, College of Agriculture and Marine Sciences, Sultan Qaboos University, Muscat, Sultanate of Oman.

${ }^{3}$ Corresponding author: jog25@cornell.edu
}

their rumination, activity, and HIS patterns around CD, alterations of blood markers of metabolic and health status around calving, reduced milk production, and were more likely to exit the herd than cows not flagged based on the HIS and cows without disease, suggesting that cows flagged based on the HIS had a more severe episode of metritis. Including all disorders of interest for this study, the overall sensitivity was $59 \%$, specificity was $98 \%$, positive predictive value was $58 \%$, negative predictive value was $98 \%$, and accuracy was $96 \%$. The AHMS was effective for identifying cows with severe cases of metritis, but less effective for identifying cows with mild cases of metritis. Also, the overall accuracy and timing of the AHMS alerts for cows with health disorders indicated that AHMS that combine rumination and activity could be a useful tool for identifying cows with metabolic and digestive disorders, and more severe cases of mastitis and metritis.

Key words: rumination, activity, metritis, dairy cow

\section{INTRODUCTION}

Metritis is a prevalent disease that affects lactating dairy cows in the early postpartum period. The occurrence of this health disorder, which may affect up to $40 \%$ of cows (Markusfeld, 1987; Zwald et al., 2004; Sheldon et al., 2009), has been associated with reduced milk production, impaired reproductive performance, and increased risk of culling during lactation (Bell and Roberts, 2007; Wittrock et al., 2011; Giuliodori et al., 2013). Therefore, commercial dairy farms implement health-monitoring programs to detect and treat cows with metritis in an attempt to reduce its consequences for cow performance. Although health-monitoring programs vary significantly among farms, the diagnosis of metritis is based primarily on evaluation of the uterus and uterine discharge. The presence of an abnormally enlarged uterus and a fetid, watery, pink-brown uterine discharge are considered evidence of metritis (Földi et al., 2006; Sheldon et al., 2006; 2009). In addition, signs of systemic illness such as anorexia, fever, depression, and dehydration provide information about the general status of the cow and the severity of the disease 
(Benzaquen et al., 2007; Sheldon et al., 2009; Risco and Melendez Retamal, 2011).

Beyond clinical examination and evaluation of the uterus and its contents, few diagnostic aids are available to identify cows with metritis in dairy farms. Research studies have documented moderate sensitivity (Se) and specificity (Sp) for methods such as feeding behavior (Urton et al., 2005) and blood concentrations of the acute-phase protein haptoglobin (Huzzey et al., 2009). But even though these tests may help identify cows with metritis, the greatest limitation for their widespread use in commercial dairy farms is on-farm implementation. The recent development of noninvasive sensor technologies for monitoring rumination time and physical activity could be used to assess overall cow health and aid in the detection of cows with metritis. Recent studies have documented alterations in the pattern of rumination and activity in cows diagnosed with metritis. For example, Liboreiro et al. (2015) reported lower rumination and activity after calving in cows with metritis compared with cows without metritis. Titler et al. (2013) reported that cows with metritis spent more time standing, took fewer steps, and had fewer lying bouts from 1 to $3 \mathrm{~d}$ before and after diagnosis than cows without a health disorder. Although results from these studies suggested that automated rumination and activity monitoring systems could be used to identify cows with metritis, additional data are needed to support their use in commercial dairy operations. In addition, little is known about the patterns of rumination and activity around the timing of clinical diagnosis of metritis. A better understanding of the changes in these parameters in cows with metritis may help improve the ability of farms to detect cows that are at risk or that already have the disorder. In addition, rumination and activity may offer unique insights into the overall health status of cows that are not provided by clinical examination and evaluation of the uterus.

We hypothesized that an automated health-monitoring system (AHMS) that continuously monitors rumination and activity would be able to identify cows with metritis. Also, we expected that changes in rumination and activity before evident clinical signs of disease would result in earlier identification of metritis. Thus, the objectives of this study were to evaluate (1) the performance of an automated rumination and physical activity monitoring system to identify cows with metritis; (2) the interval between the AHMS alert based on a health index score (HIS) and the day of clinical diagnosis (CD) by farm personnel; and (3) the rumination, activity, HIS, and milk-production patterns for cows with metritis. We also used culling dynamics, reproductive outcomes for first AI service, and markers of energy status [nonesterified fatty acids (NEFA) and BHB], mineral status (total calcium), and systemic inflammation (haptoglobin) to complement the diagnosis of metritis and the performance of the AHMS alert.

\section{MATERIALS AND METHODS}

\section{Animals, Management, and Study Design}

All procedures were approved by the Institutional Animal Care and Use Committee of Cornell University. The study followed an observational prospective cohort design. Details about animal management and study design are provided in a companion manuscript (Stangaferro et al., 2016a). Briefly, Holstein cows ( $\mathrm{n}=$ 1,121; 451 nulliparous and 670 multiparous) were fitted with a neck-mounted electronic rumination and activity monitoring tag (HR Tags; SCR Dairy, Netanya, Israel) to monitor rumination and activity from at least $21 \mathrm{~d}$ before expected calving until at least $80 \mathrm{~d}$ after calving. Of 1,121 cows enrolled in the study, 41 (3.7\%) were removed from the data set due to tag malfunction or misplacement during data collection. Thus, 1,080 cows were included in the final data set for analysis. Based on rumination and activity data, an HIS (0 to 100 arbitrary units) for each cow was generated by the system software (DataFlow, Netanya, Israel) using a series of internal algorithms (proprietary to SCR Dairy). An HIS of 100 arbitrary units represents a cow with an ideal pattern of rumination and activity; an HIS value $<86$ arbitrary units may be indicative of a health disorder. An HIS report was generated daily to include cows with $<86$ arbitrary units (as determined by SCR) and stored for evaluation by the research group. During the study, farm personnel did not have access to the HIS report or any information generated by the AHMS.

\section{Fresh Cow Monitoring Program and Case Definitions}

Disease definitions and the health-monitoring program used in the study are provided in detail in a companion manuscript (Stangaferro et al., 2016a). In short, after calving, cows were examined daily from 1 to 10 DIM. The clinical examination included direct observation, rectal temperature, urine ketones, and rumen auscultation. For any cow not diagnosed with metritis before 8 DIM, transrectal massage of the uterus was conducted to obtain and evaluate uterine discharge. Metritis was defined as the presence of watery, pink/ brown, and fetid uterine discharge with or without fever (rectal temperature $\geq 39.5^{\circ} \mathrm{C}$ ).

Two consecutives episodes of metritis were considered separate episodes if they occurred at least $7 \mathrm{~d}$ apart. 


\section{Blood Collection and Laboratory Analyses}

Briefly, blood samples were collected from a subgroup of cows $(\mathrm{n}=459)$ on $\mathrm{d}-11 \pm 3$ and $-4 \pm 3$ prepartum, and then at $0,3 \pm 1,7 \pm 1,14 \pm 1$, and $28 \pm 1 \mathrm{~d}$ after calving. Plasma samples were analyzed for NEFA (d -11, -4, 0, 3, 7, 14, 28), BHB (d 0, 3, 7, 14, 28), total Ca (d 0, 3, 7, 14), and haptoglobin (d -4, $0,3,7,14,28)$. Details about blood collection, blood sample processing, and analysis of metabolites (NEFA, BHB), calcium, and markers of systemic inflammation (haptoglobin) are provided in a companion manuscript (Stangaferro et al., 2016a).

\section{Statistical Analysis}

System Performance. The main outcome of interest for this study was the ability of the HIS to correctly identify cows with metritis. Clinical diagnosis by farm personnel was used as the reference test. Because HIS does not confirm the occurrence of disease or indicate the type of disease, a positive outcome was defined as an HIS value of $<86$ arbitrary units during at least $1 \mathrm{~d}$ during the $5 \mathrm{~d}$ before, the day of, or $2 \mathrm{~d}$ after CD. The Se and $95 \%$ CI for the HIS to identify cows with metritis was calculated using PROC FREQ in SAS (version 9.4; SAS Institute Inc., Cary, NC) and was defined as the ability of the HIS to correctly identify cows diagnosed with metritis by farm personnel. To evaluate the potential confounding effect of other health disorders (i.e., all disorders of interest, pneumonia, and lameness) on the Se of HIS, we conducted 3 separate analyses. The first analysis included all cows diagnosed with metritis; a second analysis included cows diagnosed only with metritis during the range of interest around CD; and a third analysis included cows diagnosed with metritis and at least one other health disorder during the range of interest around CD. Unfortunately, uterine discharge characteristics (appearance, color, and odor), severity of metritis, or both, based on clinical signs, were not recorded. Thus, in an attempt to identify cows with metritis cases of different severity, we also calculated the Se of HIS for subgroups of cows with metritis created based on rectal temperature at the time of CD. For this analysis, cows were included in 1 of the following rectal temperature groups: $(1) \leq 39.4^{\circ} \mathrm{C}$, no fever; (2) 39.5 to $39.9^{\circ} \mathrm{C}$, moderate fever; and $(3) \geq 40^{\circ} \mathrm{C}$, high fever. We determined differences in Se of HIS between the subgroup of cows with metritis only and cows with metritis and at least another disorder, and for the different rectal temperature subgroups, by logistic regression using the events over trials option of PROC LOGISTIC in SAS.
Interval Between the First Positive HIS Outcome and Clinical Diagnosis. To determine if HIS was capable of identifying cows with metritis earlier than CD by farm personnel, the interval (in days) between the first positive HIS outcome during the period of interest around CD ( $5 \mathrm{~d}$ before to $2 \mathrm{~d}$ after) and the day of CD of metritis was evaluated. For this analysis, which included only cows flagged by the AHMS, we compared the mean number of days from the first HISpositive outcome and the day of CD with a paired $t$-test using the PROC TTEST in SAS.

Rumination, Activity, HIS, and Milk Production Relative to Clinical Diagnosis. Daily rumination time $(\mathrm{min} / \mathrm{d})$, daily activity (arbitrary units/d), and HIS (arbitrary units) were evaluated from $5 \mathrm{~d}$ before to $5 \mathrm{~d}$ after $\mathrm{CD}(\mathrm{d} 0)$ for the first event of metritis. Milk production $(\mathrm{kg} / \mathrm{d})$ was evaluated only from $5 \mathrm{~d}$ before until the day of $\mathrm{CD}$, because data were not available after CD for cows treated with an antibiotic that required milk withdrawal. Before statistical analysis, we assessed normality of the data for rumination, activity, HIS, and milk production using the Shapiro-Wilk statistic and graphical methods (histogram and Q-Q plot) and using PROC UNIVARIATE in SAS. Based on this analysis, no data transformations were necessary.

For this analysis, cows were grouped according to the following criteria: CD-positive and HIS-positive $(\mathbf{H I}+$; HIS $<86$ arbitrary units at least $1 \mathrm{~d}$ within 5 $\mathrm{d}$ before, the day of, and $2 \mathrm{~d}$ after $\mathrm{CD}$ ); CD-positive and HIS-negative (HI-; HIS $\geq 86$ arbitrary units from $5 \mathrm{~d}$ before, the day of, and $2 \mathrm{~d}$ after CD); and CDnegative (nondisease, ND; cows not diagnosed with a health disorder during the study period). For cows in the ND group, we considered the average DIM at CD for cows that had metritis "day 0." Data was analyzed by ANOVA with repeated measurements using PROC MIXED in SAS. Models for each outcome of interest (rumination, activity, HIS, and milk production) included group (e.g., ND, HI+, and HI-), time, parity, and the interactions between group and time, and group and parity, as explanatory variables. The occurrence of another health disorder (i.e., all disorders of interest, pneumonia, and lameness) during the -5 to $5 \mathrm{~d}$ period after $\mathrm{CD}$ was offered as a categorical variable $(0=$ no occurrence and $1=$ occurrence) to the initial models to evaluate the potential effect of multiple disorders on the parameters of interest. Cow within group was included as a random effect in all models. The final model for each parameter of interest was selected by backward elimination of explanatory variables with $P>0.10$ and determination of the lowest value for the Akaike's information criterion. Cow was the subject of the repeated measurements, and all 
models were run using an autoregressive (AR-1) covariance structure. Group, time, and the group-by-time interaction were forced in all models. When the main effect or interaction between explanatory variables was significant, we used the LSD post hoc mean separation test to determine differences between groups of means.

Plasma Concentration of NEFA, BHB, Calcium, and Haptoglobin. Cows were grouped in the same way as for evaluation of rumination, activity, and HIS. Data were analyzed by ANOVA with repeated measurements using PROC MIXED in SAS as described.

Culling Dynamics and Reproductive Outcomes for First AI Service. The proportion of cows coded as do not breed, sold, and dead within the first 60 DIM or up to 270 DIM were analyzed by logistic regression using PROC GLIMMIX in SAS. The proportion of cows inseminated after a detected estrus or timed $\mathrm{AI}$, and pregnancies per AI after first AI service were also analyzed by logistic regression using PROC GLIMMIX in SAS. The regression models included group $(\mathrm{HI}+, \mathrm{HI}-$, and ND), parity, and their interaction as explanatory variables. The final model for each variable of interest was selected by backward elimination of explanatory variables with $P>0.10$. The effect of group (HI+, HI-, and ND) was forced in all models. We used the LSD post hoc mean separation test was used to determine differences between groups of means. Differences in DIM at first service were analyzed by ANOVA using PROC MIXED in SAS and models that included the same explanatory variables used for the binomial outcomes.

Overall System Performance. Because HIS is designed as an alert to identify cows for further clinical examination rather than to provide a definitive diagnosis of a health disorder, false-positive outcomes could not be assigned to a particular health disorder, because the reason for the alert was unknown. Therefore, we calculated Sp, positive predictive value (PPV), negative predictive value (NPV), and accuracy as an overall test for all events recorded for the disorders of interest [metabolic and digestive (Stangaferro et al., 2016a), mastitis (Stangaferro et al., 2016b), and metritis (this paper)]. Because each day was considered a new test, the total contribution of individual cows to the number of cow-days was determined from 2 to 80 DIM. A cow could contribute with a positive or negative outcome during specific periods of the study depending on her clinical status (ND vs. with a disorder) as defined by CD. Cows that were sold or died before the end of the study did not contribute to the cow-day calculation after they left the herd.
We created a $2 \times 2$ frequency table based on the following criteria:

- True positive $=$ positive HIS outcome (HIS $<86$ arbitrary units) at least $1 \mathrm{~d}$ within the $5 \mathrm{~d}$ before, the day of, and $2 \mathrm{~d}$ after CD. Each day within the range of interest was counted as a true positive outcome when HIS was $<86$ arbitrary units for at least $1 \mathrm{~d}$ within the range of interest.

- False positive = positive HIS outcome for a cow without a health disorder or positive HIS outcome outside the -5 to $2 \mathrm{~d}$ range relative to $\mathrm{CD}$ for cows with health disorders.

- True negative = negative HIS outcome (HIS $\geq 86$ arbitrary units) for a cow without a health disorder or negative HIS outcome outside of the -5 to $2 \mathrm{~d}$ range relative to $\mathrm{CD}$ for cows with health disorders.

- False negative = negative HIS outcome during the -5 to $2 \mathrm{~d}$ range relative to $\mathrm{CD}$ for a cow with a health disorder. Each day within the range of interest was counted as a false negative outcome.

This frequency table was then used to estimate the overall Se, Sp, PPV, NPV, and accuracy of the HIS using PROC FREQ of SAS. Only the diseases of interest (displaced abomasum, ketosis, indigestion, mastitis, and metritis) could contribute as a positive disease outcome (true positive or false negative), because the window from -5 to $2 \mathrm{~d}$ relative to $\mathrm{CD}$ was removed for health disorders of no interest to the study (pneumonia and lameness).

Data for proportions are presented as arithmetical means and their 95\% CI; quantitative data are presented as LSM \pm SEM or 95\% CI, unless otherwise stated. All explanatory variables and their interactions were considered significant if $P \leq 0.05$, and $0.05<P \leq$ 0.10 was considered a tendency.

\section{RESULTS}

\section{Metritis Incidence and System Performance}

Table 1 summarizes the incidence of metritis, DIM at $\mathrm{CD}$, and Se of HIS to identify cows with metritis for all cows with a recorded case of metritis, cows diagnosed with metritis only, and cows diagnosed with metritis and another health disorder from -5 to $2 \mathrm{~d}$ after CD. Data are also presented for subgroups of cows with metritis and fever or no fever at the time of CD. The overall incidence of metritis was $32 \%$ ( $\mathrm{n}=349 ; 331$ and 9 cows with 1 or 2 events of metritis recorded, 
Table 1. Incidence of metritis, DIM at clinical diagnosis, sensitivity of health index score (HIS) to detect cows with metritis, and interval between the first HIS-positive outcome and clinical diagnosis (CD) of metritis by farm personnel

\begin{tabular}{|c|c|c|c|c|c|c|c|c|}
\hline Item & $\begin{array}{c}\text { Events } \\
(\text { no. })^{1}\end{array}$ & $\begin{array}{c}\text { Incidence } \\
(\%)\end{array}$ & $\begin{array}{l}\text { DIM at event } \\
(\text { mean } \pm \text { SD })\end{array}$ & \multicolumn{2}{|c|}{ Sensitivity } & \multicolumn{3}{|c|}{ HIS-positive to $\mathrm{CD}^{2}$} \\
\hline Metritis $^{3}$ & 349 & 32 & $6.8 \pm 2.6$ & $55(191 / 349)$ & 49,60 & -1.2 & $-1.6,-0.7$ & $<0.01$ \\
\hline Metritis only ${ }^{4}$ & 322 & 30 & $6.8 \pm 2.6$ & $53(170 / 322)^{\mathrm{a}}$ & 47,58 & -1.2 & $-1.6,-0.7$ & $<0.01$ \\
\hline Metritis with other disorders ${ }^{5}$ & 27 & 2 & $7.0 \pm 2.4$ & $78(21 / 27)^{\mathrm{b}}$ & 62,91 & -1.3 & $-2.4,-0.2$ & 0.03 \\
\hline \multicolumn{9}{|l|}{ Metritis by rectal temperature } \\
\hline$\leq 39.4^{\circ} \mathrm{C}$ & 165 & 52 & $7.2 \pm 1.8$ & $56(92 / 165)$ & 48,64 & -1.4 & $-1.9,-1.0$ & $<0.01$ \\
\hline
\end{tabular}

${ }^{\mathrm{a}, \mathrm{b}}$ Different superscripts indicate differences $(P \leq 0.05)$ between means based on mean separation with the LSD test.

${ }^{1}$ Number of events diagnosed (331 and 9 cows with 1 or 2 events of metritis, respectively).

${ }^{2}$ HIS-positive to $\mathrm{CD}=$ interval in days between the first positive HIS outcome (positive outcomes only) and clinical diagnosis.

${ }^{3}$ All metritis events recorded.

${ }^{4}$ Cows diagnosed with only metritis from -5 to $2 \mathrm{~d}$ relative to $\mathrm{CD}$

${ }^{5}$ Cows diagnosed with metritis and at least another health disorder from -5 to $2 \mathrm{~d}$ relative to CD.

respectively). The overall Se of HIS to identify cows with metritis was $55 \%$, and it was greater $(P=0.02)$ for cows with metritis and another health disorder than for cows with metritis only. Conversely, the Se of HIS did not differ $(P=0.52)$ for subgroups of cows with or without fever. Overall, cows with metritis and a positive HIS outcome were identified earlier than CD by farm personnel.

\section{Rumination, Activity, HIS, and Milk Production Relative to Clinical Diagnosis}

Daily rumination time, activity, and HIS patterns from -5 to $5 \mathrm{~d}$ relative to $\mathrm{CD}$ for cows in the $\mathrm{HI}+$, HI-, and ND groups are presented in Figure 1. For all parameters, the effect of parity and the group-byparity interaction were not significant. For rumination, we observed an interaction between group and day $(P$ $<0.01$ ). Rumination time was lower for cows with metritis in the HI+ $(\mathrm{n}=187)$ than in the HI- $(\mathrm{n}=153)$ or ND groups $(\mathrm{n}=435)$; however, it was similar for the HI- and ND groups over the entire period. Because metritis was diagnosed on average at $\sim 7$ DIM, the nadir for rumination for all groups $(313,376$, and 368 $\mathrm{min} / \mathrm{d}$ for the $\mathrm{HI}+, \mathrm{HI}-$, and ND groups) was observed on $\mathrm{d}-5(\sim 2$ DIM average $)$. Thereafter, rumination increased for all groups, but the increment was slower for cows in the HI+ group, and faster for cows in the HI- and ND groups. We observed the greatest difference for rumination between cows in the HI+ group and cows in the HI- (122 $\mathrm{min} / \mathrm{d})$ and ND $(131 \mathrm{~min} / \mathrm{d})$ groups $1 \mathrm{~d}$ before CD. Rumination time for cows with metritis was also affected by the occurrence of ketosis, because cows with ketosis had reduced rumination $(P$ $=0.01)$ than cows without ketosis during the period of interest around CD $(407 \pm 16.1$ vs. $452 \pm 2.1 \mathrm{~min} / \mathrm{d}$, respectively).

For activity, we observed an interaction between group and day $(P<0.01)$. Cows in the HI+ group had lower activity than cows in the HI- and ND groups during the entire period evaluated, reaching their nadir (434 arbitrary units/d) on d 0; cows in the HI- group had lower activity than cows in the ND group on $\mathrm{d}-5$. Activity for cows with metritis was also affected $(P=$ 0.03 ) by the occurrence of ketosis. Cows with ketosis had less activity than cows without ketosis during the period of interest around CD (467 \pm 32.6 vs. $534 \pm 4.1$ arbitrary units/d, respectively).

For HIS, we also detected an interaction between group and day $(P<0.01)$. Cows in the HI+ group had lower HIS than cows in the HI- and ND groups, but cows in the HI- and ND group had similar HIS during the entire period analyzed. The HIS for cows in the $\mathrm{HI}+$ group reached its lowest value $(83$ units) on $\mathrm{d} 0$. The HIS was also affected by the occurrence of ketosis, because cows with metritis and ketosis had lower HIS $(P=0.04)$ than cows without ketosis during the period of interest around CD $(90 \pm 1.7$ vs. $95 \pm 0.2$ arbitrary units, respectively).

Daily milk production from $5 \mathrm{~d}$ before until the day of $\mathrm{CD}$ for cows with metritis, grouped based on HIS outcome (HI+ and $\mathrm{HI}-$ ), is presented in Figure 2. Because parity had an effect $(P<0.01)$ on milk production, results are presented separately for primiparous and multiparous cows. For primiparous cows (Figure $2 \mathrm{~A}$ ), we observed an interaction between group and day $(P<0.01)$. Cows in the HI+ group produced less milk than cows in the ND group from $4 \mathrm{~d}$ before until the day of CD; cows in HI+ group produced less milk than cows in the HI- group on $\mathrm{d}-2$ and 0 relative to CD. 


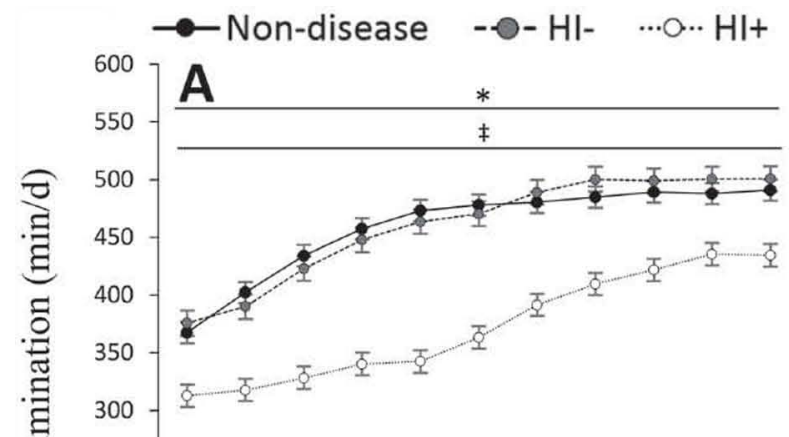

The greatest difference between cows in the $\mathrm{HI}+$ and cows in the HI- and ND groups was observed -2 and 0 d relative to $\mathrm{CD}$ (2.4 and $3.2 \mathrm{~kg} / \mathrm{d}$, respectively). For multiparous cows (Figure 2B), we observed an interaction between group and day $(P<0.01)$. Multiparous cows in the HI+ group produced less milk than cows in the HI- and ND groups from $4 \mathrm{~d}$ before until the day of $\mathrm{CD}$, with the greatest differences -1 and $0 \mathrm{~d}$ relative to CD of metritis $(\sim 7 \mathrm{~kg} / \mathrm{d})$. Cows in the HI- and ND groups produced similar amounts of milk during the period analyzed.

\section{Plasma Concentrations of NEFA, BHB, Calcium, and Haptoglobin}

Plasma concentrations of NEFA, BHB, Ca, and haptoglobin relative to the day of calving for cows that developed metritis are presented in Figure 3. For NEFA, we observed an interaction between group and day $(P<0.01)$. Plasma concentrations of NEFA were greater in the $\mathrm{HI}+$ than in the ND group from -11 to $7 \mathrm{~d}$ relative to calving. Cows in the HI- group had lower NEFA concentrations than cows in the HI+ group 0 and $3 \mathrm{~d}$ relative to calving, and we observed no differences with the ND group during the period analyzed. Concentrations of NEFA peaked on d 0 (488 $\pm 24 \mu \mathrm{Eq} / \mathrm{L}$ ) for cows in the HI+ group and differed by 85 and $126 \mu \mathrm{Eq} / \mathrm{L}$ compared with cows in the ND and HI- group, respectively. For BHB, we observed an effect of day $(P=0.04)$ and a tendency for an interaction between group and day $(P=0.08)$. In addition, BHB concentrations were also affected $(P<0.01)$ by the occurrence of displaced abomasum. Cows with a displaced abomasum had greater BHB concentrations than cows without $(13.4 \pm 1.5$ vs. $7.6 \pm 0.1 \mathrm{mg} / \mathrm{dL}$, respectively). For concentrations of total calcium in plasma, we observed an interaction between group and day $(P<0.01)$. Cows in the HI+ group had total calcium concentrations that were lower than the ND group from 3 to $14 \mathrm{~d}$ after calving, and lower than the HI- group from 7 to $14 \mathrm{~d}$ after calving. Cows in the $\mathrm{HI}+$ group had their lowest calcium concentration (8.4 $\mathrm{mg} / \mathrm{dL}$ ) on $\mathrm{d} 3$ after calving, and the greatest difference with cows in the HI- $(0.5 \mathrm{mg} / \mathrm{dL})$ and ND group (0.6 $\mathrm{mg} / \mathrm{dL}) 14 \mathrm{~d}$ after calving. Cows in the HI- and ND group had similar calcium concentrations during the entire period analyzed. For concentrations of haptoglobin, we observed an interaction between group and day $(P<0.01)$. Cows in the HI+ group presented greater haptoglobin concentrations than cows in the ND group from 0 to $14 \mathrm{~d}$ after calving reaching peak concentrations $(4.0 \mathrm{mg} / \mathrm{mL})$ and the greatest difference with the ND group $(2.1 \mathrm{mg} / \mathrm{mL}) 3 \mathrm{~d}$ after calving. Cows in the 

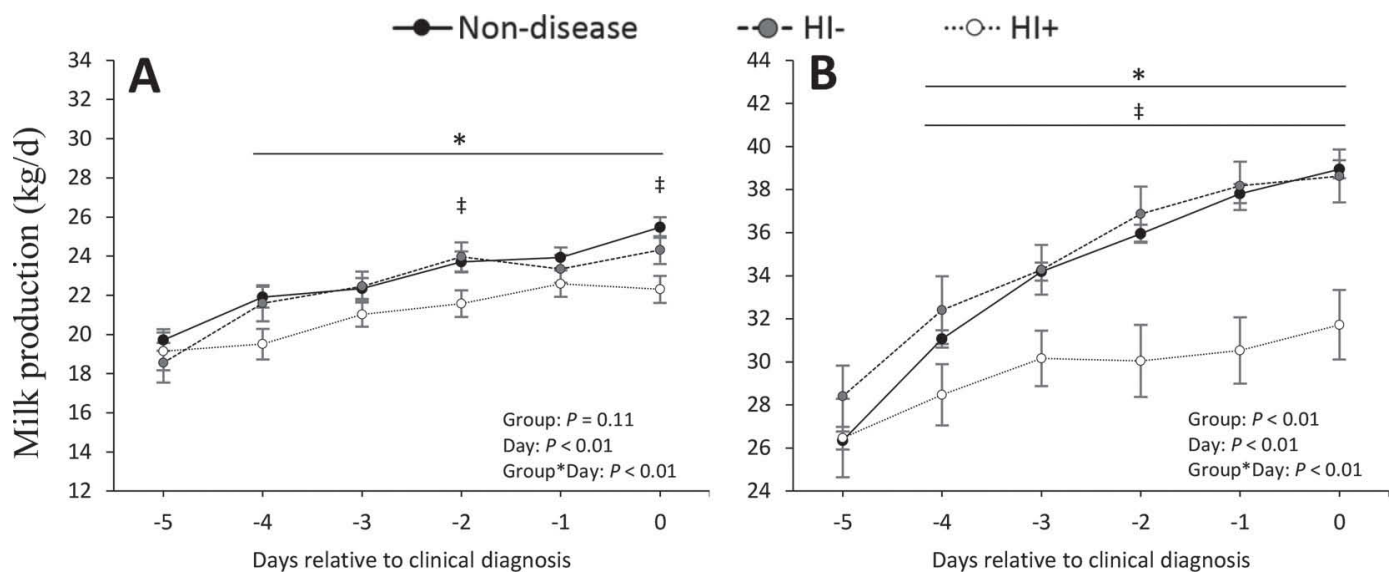

Figure 2. Milk production $(\mathrm{kg} / \mathrm{d})$ from $-5 \mathrm{~d}$ to the day of clinical diagnosis (CD; d 0 ) for primiparous cows (A) that developed metritis $(\mathrm{HI}+: \mathrm{n}=110 ; \mathrm{HI}-: \mathrm{n}=96)$ compared with nondisease cows $(\mathrm{n}=171)$. Cows were assigned to the HI+ or HI- group if they had a health index score of $<86$ or $\geq 86$ arbitrary units, respectively, during the $5 \mathrm{~d}$ before, the day of, and $2 \mathrm{~d}$ after $\mathrm{CD}$. Milk production (kg/d) from $-5 \mathrm{~d}$ to the day of CD for multiparous cows (B) that developed metritis compared with nondisease cows $(\mathrm{n}=264)$. Multiparous cows with metritis were assigned to the HI+ $(\mathrm{n}=77)$ and $\mathrm{HI}-(\mathrm{n}=57)$ groups following the same criteria as for primiparous cows with metritis. Values are presented as LSM \pm SEM. Within a day, pairwise comparisons that were statistically different $(P \leq 0.05)$ based on LSD are represented as follows: *control vs. HI+; $\ddagger \mathrm{HI}+$ vs. HI-.

HI- group presented greater haptoglobin concentrations than cows in the ND group from 0 to $7 \mathrm{~d}$ after calving (greatest difference $1.3 \mathrm{mg} / \mathrm{mL}$ on $\mathrm{d} 3$ ), but lower than cows in the HI+ group from 3 to $14 \mathrm{~d}$ after calving.
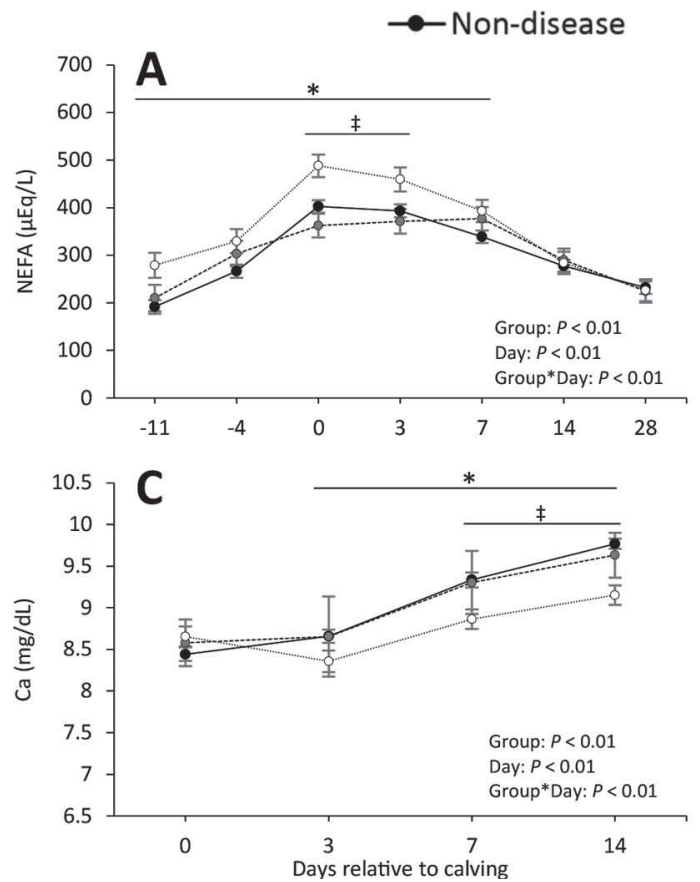

\section{Culling Dynamics and Reproductive Outcomes for First Al Service}

The proportion of cows dead up to 60 DIM $(P=$ $0.74)$ or up to 270 DIM $(P=0.33)$ was similar for cows

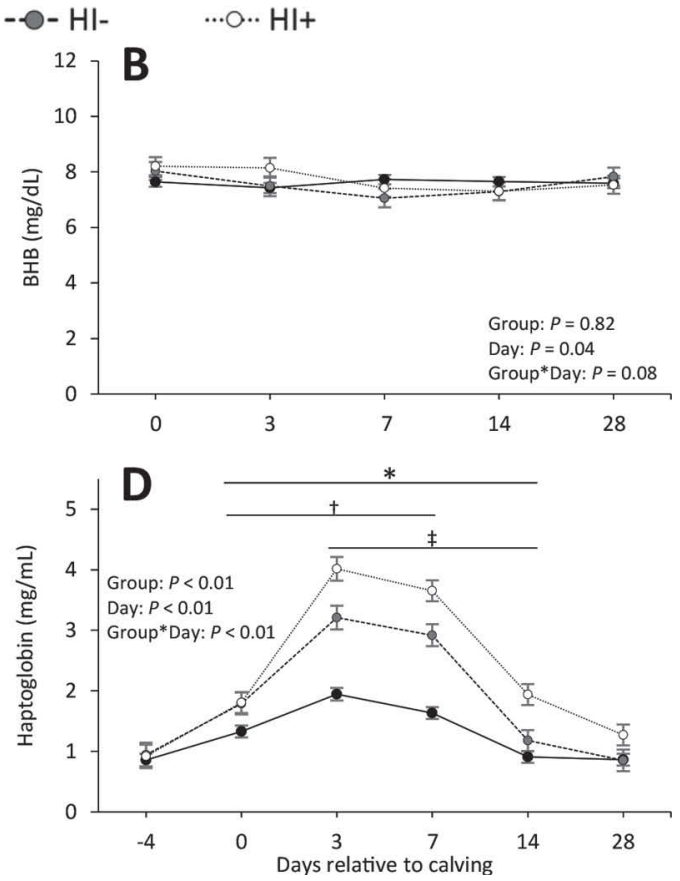

Figure 3. Plasma concentrations of nonesterified fatty acids (NEFA; A), BHB (B), total Ca (C), and haptoglobin (D) for cows that developed metritis compared with cows in the nondisease group $(\mathrm{n}=161)$. Cows with metritis were assigned to the HI+ $(\mathrm{n}=51)$ or HI- $(\mathrm{n}=48)$ group if they had a health index score of $<86$ or $\geq 86$ arbitrary units, respectively, during the $5 \mathrm{~d}$ before, the day of, and $2 \mathrm{~d}$ after clinical diagnosis. Values are presented as LSM \pm SEM. Within a day, pairwise comparisons that were statistically different $(P \leq 0.05)$ based on LSD are represented as follows: *control vs. HI+; †control vs. HI-; $\ddagger$ HI + vs. HI-. 
Table 2. Culling dynamics and reproductive outcomes for first AI service for cows diagnosed with metritis included in the ND, HI-, and HI+ groups $^{1}$

\begin{tabular}{|c|c|c|c|c|}
\hline Item & ND & HI- & $\mathrm{HI}+$ & $P$-value \\
\hline Dead up to 270 DIM [\% (no./no.)] & $2.9(13 / 451)$ & $0.7(1 / 153)$ & $2.1(4 / 187)$ & 0.33 \\
\hline Do not breed/sold $\leq 60$ DIM $[\%$ (no./no.) & $2.5(11 / 451)^{\mathrm{a}}$ & $3.3(5 / 153)^{\mathrm{a}}$ & $7.0(13 / 187)^{\mathrm{b}}$ & 0.03 \\
\hline Cows AI to estrus $[\%$ (no./no.)] & $91.7(367 / 400)$ & $91.4(128 / 140)$ & $89.8(141 / 157)$ & 0.76 \\
\hline Cows AI by timed AI [\% (no./no.)] & $8.3(33 / 400)$ & $8.6(12 / 140)$ & $10.2(16 / 157)$ & 0.76 \\
\hline DIM at first AI & $79(400)$ & $79(140)$ & $80(157)$ & 0.73 \\
\hline
\end{tabular}

${ }^{\mathrm{a}, \mathrm{b}}$ Different superscripts within a row indicate differences $(P \leq 0.05)$ between means based on mean separation with the LSD test.

${ }^{1} \mathrm{ND}(\mathrm{n}=451)=$ cows not diagnosed with a health disorder during the study period; HI $-(\mathrm{n}=153)=$ health index score $\geq 86$ arbitrary units during the $5 \mathrm{~d}$ before, the day of, or $2 \mathrm{~d}$ after clinical diagnosis; HI+ $(\mathrm{n}=187)=$ health index score $<86$ arbitrary units during at least $1 \mathrm{~d}$ during the $5 \mathrm{~d}$ before, the day of, or $2 \mathrm{~d}$ after clinical diagnosis.

in the HI+, HI-, and ND groups (Table 2). In contrast, the proportion of cows coded as do not breed or sold up to 60 DIM (end of the voluntary waiting period), as well as up to 270 DIM, were greater $(P<0.05)$ for the $\mathrm{HI}+$ than the HI- and ND groups. No differences in the proportion of cows inseminated based on a detected estrus or timed AI $(P=0.76)$, DIM at first AI $(P=$ $0.73)$, or pregnancies per AI $(P=0.80)$ among groups were detected.

\section{Overall System Performance}

From 2 to 80 DIM, the overall Se of HIS to detect all the health disorders of interest for this study (displaced abomasum, ketosis, indigestion, mastitis, and metritis) was $59.0 \%$ (95\% CI: $57.5,60.5 \%$; 2,416/4,096 cow-days) and Sp was $97.6 \%$ (95\% CI: 97.5, 97.7\%; 70,695/72,423 cow-days). Based on the prevalence observed in the study, the PPV was $58.3 \%$ (95\% CI: 56.8, 59.8\%; $2,416 / 4,144$ cow-days), the NPV was $97.7 \%$ (95\% CI $97.6,97.8 \% ; 70,695 / 72,375$ cow-days), and accuracy was $95.6 \%$ (95\% CI: 95.4, 95.7\%; 73,111/76,519 cowdays).

\section{DISCUSSION}

In the current study, we evaluated the performance of an AHMS that combined rumination and activity to identify cows with metritis. The overall Se of the AHMS was moderate and slightly lower for cows diagnosed with metritis only during the -5 to $2 \mathrm{~d}$ range relative to CD. Conversely, the Se of HIS was substantially greater (25 percentage points) for cows with metritis and another health disorder during the window of interest around CD. The incidence of metritis in our study was within the upper level of the range reported for lactating dairy cows (Bell and Roberts, 2007; Sheldon et al., 2009; Toni et al., 2015), likely because the farm standard operating procedures for diagnosis and treatment of metritis determined that cows with very mild watery, foul smelling, pink/brown discharge should be treated. Therefore, we speculated that the major reason for the Se of HIS observed in our study was a wide range in severity of the disorder, which, in turn, caused a wide range of alterations to the rumination and activity patterns around CD. Thus, we evaluated the performance of the AHMS for subgroups of cows in an attempt to identify different levels of severity. In this regard, the greater Se of HIS for cows that experienced metritis along with another disorder supports the notion that the AHMS was capable of detecting cows in a poorer health state. Moreover, when HIS group (HI+ and $\mathrm{HI}-$ ) was used as a proxy for severity and systemic compromise, cows in the HI+ group had more dramatic reductions in rumination and activity, and a greater reduction in milk production around the time of metritis CD than cows in the HI- and ND groups. For example, milk production before $\mathrm{CD}$ was lower in primiparous and multiparous cows in the HI+ group than in the ND group, but cows in the HI- group did not have decreased milk production. Moreover, the differences observed for NEFA, Ca, and haptoglobin around calving indicated that cows in the HI+ group were in a more negative plane of energy balance, were more Ca deficient, and presented more severe systemic inflammation than cows in the HI- group. The lack of difference in BHB concentrations was unexpected because of the differences for the other blood markers and the results of previous studies, which have reported an association between BHB concentrations and the risk of metritis (Duffield et al., 2009; Galvão et al., 2010; Ospina et al., 2010). Nevertheless, in agreement with the current results, other studies have not reported differences in BHB concentrations between cows with or without metritis (Chapinal et al., 2011; Martinez et al., 2012). For cows in the HI- group, intermediate 
haptoglobin concentrations suggested that they underwent systemic inflammation, but that the degree of inflammation was likely lower than for cows in the HI+ group. Furthermore, cows in the HI+ group were about twice as likely to exit the herd or be coded as do not breed (up to 60 DIM and up to 270 DIM) than cows in the HI- and ND groups. The differences in culling dynamics also help explain the lack of differences in first service reproductive performance, because many cows expected to have poor fertility and production left the herd before their first AI service. Taken together, these observations suggest that not only were cases of metritis for cows in the HI+ group more severe, but also that these cows did not cope as well with the disease, leading to their earlier removal from the herd.

Rectal temperature was also explored as a potential indicator of severity of metritis, because fever may be an indication of infection and inflammation (Mackowiak et al., 1997; Leon, 2002; Steiner et al., 2006). Nevertheless, the Se of the AHMS did not increase substantially for cows with fever compared with that of cows without fever, suggesting that in this case fever was not a good marker of severity. This was not surprising, because a previous study (Benzaquen et al., 2007 ) reported that up to $58 \%$ of lactating dairy cows diagnosed with metritis did not develop fever (defined as rectal temperature of $\geq 39.4^{\circ} \mathrm{C}$ ).

Collectively, our findings seem to support the notion that there are varying levels of severity and systemic compromise for metritis, and that the ability of the HIS generated by the AHMS to identify cows with metritis depends upon the degree to which overall cow health and behavior are compromised. Based on our current limited knowledge of the physiological effect of metritis on rumination and physical activity, we speculate that the patterns of these parameters change more drastically when the uterine infection affects cows systemically rather than when it affects the uterus only. Additional research studies that include objective definitions of severity and systemic compromise for cases of metritis are needed to corroborate our observations and continue improving algorithms used to identify cows with metritis.

For cows with metritis detected based on HIS, the AHMS identified them earlier than farm personnel. This is in agreement with our data for cows diagnosed with metabolic and digestive disorders (Stangaferro et al., 2016a) and cows diagnosed with mastitis, which were also identified earlier based on HIS than by farm personnel (Stangaferro et al., 2016b). The earlier identification of cows based on HIS was expected, because cows in the HI+ group presented substantial reductions in rumination and activity on the days leading up to
CD. The value of identifying cows with metritis approximately $1 \mathrm{~d}$ earlier based on HIS than with traditional health-monitoring programs is currently unknown. Earlier treatment could be beneficial by halting the progression of the disorder and improving treatment response. Earlier treatment could also help mitigate the negative effect of the disease on milk production and reduce the likelihood of culling. Additional studies are necessary to determine the value of earlier treatment on cow well-being and performance during lactation.

The pattern of rumination and activity for cows with metritis reflected the timing of metritis diagnosis during lactation. Dairy cattle have a rumination nadir on the day of calving (Soriani et al., 2012; Calamari et al., 2014; Liboreiro et al., 2015), followed by a sharp increase until a plateau is reached at $\sim 7$ DIM. Activity patterns are also profoundly altered around calving, but a sharp increase rather than a decrease is usually observed. The peak observed on the day of calving is followed by a steady decline up to $\sim 6$ DIM, after which activity remains steady (Liboreiro et al., 2015; M. L. Stangaferro and J. O. Giordano, unpublished results). These expected patterns of rumination and activity in dairy cows explain the general trends observed for all cows regardless of health status. For cows in the ND group, $\mathrm{d}-5$ corresponded to an average of 2 DIM because $\mathrm{d} 0$ for this group was equal to the mean DIM for diagnosis of metritis (6.8 DIM). For cows diagnosed with metritis, $\mathrm{d}-5$ included some cows that were as early as 1 DIM because the earliest time of metritis diagnosis was 3 DIM. Regardless of the influence of DIM on the pattern of rumination and activity, cows affected by metritis showed differences compared to cows without a health disorder. Liboreiro et al. (2015) have also reported reduced rumination time and activity within the first week after calving in lactating dairy cows diagnosed with metritis.

From an on-farm implementation perspective, the results of the current experiment suggest that the HIS generated by the AHMS tested in our study could be used to detect severe cases of metritis that affect the cow systemically or cases of metritis accompanied by another health disorder. However, the most likely scenario is that not all cows that develop metritis will be detected. This is because the underlying health disorder may not compromise cow health sufficiently to affect rumination and activity. Thus, it seems reasonable to suggest that for farms with proactive health-monitoring and treatment programs, the AHMS could be used in combination with other traditional methods of metritis detection. For example, the AHMS could be used to identify cows with severe cases of metritis up to 7 to 10 DIM when most cases are expected (Sheldon et al., 
2009; current study). Thereafter, cows not diagnosed with metritis could be examined to rule out the presence of the disorder. For cows with metritis detected during the mandatory examination (mild cases without a significant systemic compromise), a minor delay from the onset of the disorder to diagnosis may not comprise cow well-being, the outcome of the treatment, or the future productive and reproductive performance of the cow. Research experiments should be conducted to test the feasibility of on-farm implementation of these types of strategies. Unlike farms with proactive health-monitoring programs, farms that do not actively examine cows to detect metritis may benefit from using rumination and activity to detect cows with the most severe cases of the disorder. In this case, it is also unlikely that cows with mild or less severe cases would be identified by farm personnel because of the lack of a systematic health-monitoring program.

The HIS generated by the AHMS presented high accuracy when all the disorders of interest (displaced abomasum, ketosis, indigestion, metritis and mastitis) were included in the analysis. This is likely a reflection of the high Sp and NPV (both $\geq 97 \%$ ) and the considerably greater number of cow-days during which cows did not have a health disorder $(\mathrm{n}=72,423)$ rather than when they did have a health disorder $(\mathrm{n}=4,096)$. These observations also suggest that a HIS value of $\geq 86$ arbitrary units is a reasonable indicator that cows are not affected by a health disorder. Generating the fewest false-positive alerts is an important attribute of an AHMS to avoid the unnecessary inclusion of cows without a health disorder in reports created to select cows for clinical examination. Conversely, the Se and PPV for all disorders combined were close to $60 \%$, considerably lower and undesirable for on-farm implementation. The main reason, however, for the relatively low Se was the wide range of Se for the disorders monitored in the study and the considerably greater number of cows with disorders for which the HIS presented moderate Se. For all metabolic and digestive disorders combined, the overall Se was $93 \%$, and the number of health events represented only $20 \%$ of the total number recorded (Stangaferro et al., 2016a). In contrast, the overall Se for cases of metritis and mastitis was $\sim 55 \%$ (Stangaferro et al., 2016b) but they represented $80 \%$ of all health events. Two other factors also contributed to the moderate PPV. First, the restricted window around CD ( -5 to $2 \mathrm{~d}$ after $\mathrm{CD}$ ) to consider a positive outcome from the AHMS as true positive led to the misclassification of some outcomes as false positives. Indeed, $10.7 \%$ of the cows presented an HIS-positive outcome 6 to $8 \mathrm{~d}$ before $\mathrm{CD}$, and $13.8 \%$ continued to present an HIS-positive outcome 3 to $5 \mathrm{~d}$ after CD. On the other hand, a very low daily incidence of health disorders favors a lower PPV, because the presence of a few false-positive outcomes per day outweighs the number of true-positive outcomes (PPV $=$ number of true positive outcomes divided by the total number of true-positive + false-positive outcomes).

To determine the Sp of the HIS in a valid way, we eliminated data from $3.7 \%$ of the cows enrolled. In these cows the rumination and activity monitoring tag was either misplaced or malfunctioned during the study, resulting in a rumination and activity pattern characterized by consistently low values (usually $<100 \mathrm{~min} / \mathrm{d}$ of rumination or $<100$ arbitrary units/d for activity) during prolonged periods (at least $14 \mathrm{~d}$ ). Because this is a known limitation of the AHMS used in our study, specific reports including cows with a malfunctioning or misplaced tag can be created to replace the tag or place it in the correct position.

We are uncertain whether the AHMS used in our study would be equally effective in identifying cows with health disorders in other commercial dairy herds with similar management. Indeed, using CD by farm personnel as reference for the occurrence and timing of diagnosis of clinical health disorders could make our results unique to the farm where the study was conducted. Therefore, additional studies are needed to confirm our findings. However, data from the present study provides compelling evidence that automated rumination and activity monitoring systems could be incorporated either alone or in combination with other traditional health-monitoring strategies to identify cows with metabolic and digestive disorders and severe cases of clinical mastitis and metritis. For dairy herds with very intensive health-monitoring programs, such as the herd that participated in our study, some cows with health disorders may not be identified by the AHMS. Nevertheless, our current data suggest that cows not flagged based on HIS may have had a less severe episode of the disorder and experienced a less severe reduction in performance (i.e., milk production). Because rumination and activity patterns for individual cows can also be evaluated separately, under some circumstances it may be possible to identify cows for clinical examination based on rumination and activity alone, rather than by HIS. Thus, in herds with intensive health-monitoring programs, the AHMS could be used as an alternative or a complement to human monitoring. Such a strategy would also reduce the resources and time needed for health monitoring while minimizing disruption of cow normal behavior and time budgets. Conversely, for dairy farms with passive health-monitoring programs (i.e., no monitoring or minimal monitoring) the AHMS may help improve overall cow health and performance 
by identifying cows with health disorders that would not be recognized otherwise. Regardless of the level of intensity of the current health-monitoring program, all farms may benefit by early disease detection (for cows detected by the AHMS) by improving the treatment response and reducing the negative consequences of health disorders on overall cow health, productive performance, and reproductive performance. Likely, the greatest benefit would be realized in herds with passive health-monitoring programs. In such herds, detection of cows with health disorders may be important not only to optimize productivity but also to improve overall cow health and well-being.

\section{CONCLUSIONS}

Our findings demonstrated that automated rumination and activity monitoring was effective for identifying cows with severe cases of metritis or cows with metritis and another health disorder. Conversely, the ability of the AHMS to identify cows with mild cases of metritis was moderate. Overall, cows with metritis were identified earlier than through CD by farm personnel. The patterns of rumination, activity, and HIS from -5 to $5 \mathrm{~d}$ after CD for cows with metritis identified based on HIS were characterized by marked differences compared to cows in the ND group as early as $5 \mathrm{~d}$ before CD. Conversely, cows with metritis not identified based on HIS had rumination and activity patterns very similar to cows in the ND group and different from cows diagnosed with the disorder but flagged based on HIS. We conclude that automated health-monitoring systems that use rumination and physical activity should be used in combination with or to complement traditional methods of metritis detection. Future research is needed to evaluate the effect of management programs that combine rumination and activity monitoring with traditional methods to diagnose metritis on cow wellbeing and performance.

\section{ACKNOWLEDGMENTS}

The authors extend their gratitude to the owners, managers, and personnel of the commercial dairy farm that participated in the study for providing access to their cows and facilities, and their collaboration with our research team. We also extend our gratitude to SCR Dairy (Madison, WI) for partial financial and logistical support to conduct this research and to Thomas Overton (Cornell University, Ithaca, NY) for providing useful feedback on the manuscript. Matias Stangaferro received partial support from a Fulbright fellowship and from Universidad Nacional del Litoral, Esperanza, Argentina.

\section{REFERENCES}

Bell, M. J., and D. Roberts. 2007. The impact of uterine infection on a dairy cow's performance. Theriogenology 68:1074-1079.

Benzaquen, M. E., C. Risco, L. Archbald, P. Melendez, M.-J. Thatcher, and W. Thatcher. 2007. Rectal temperature, calving-related factors, and the incidence of puerperal metritis in postpartum dairy cows. J. Dairy Sci. 90:2804-2814.

Calamari, L., N. Soriani, G. Panella, F. Petrera, A. Minuti, and E. Trevisi. 2014. Rumination time around calving: An early signal to detect cows at greater risk of disease. J. Dairy Sci. 97:3635-3647.

Chapinal, N., M. Carson, T. F. Duffield, M. Capel, S. Godden, M. Overton, J. E. P. Santos, and S. J. LeBlanc. 2011. The association of serum metabolites with clinical disease during the transition period. J. Dairy Sci. 94:4897-4903.

Duffield, T. F., K. D. Lissemore, B. W. McBride, and K. E. Leslie 2009. Impact of hyperketonemia in early lactation dairy cows on health and production. J. Dairy Sci. 92:571-580.

Földi, J., M. Kulcsar, A. Pecsi, B. Huyghe, C. De Sa, J. Lohuis, P. Cox, and G. Huszenicza. 2006. Bacterial complications of postpartum uterine involution in cattle. Anim. Reprod. Sci. 96:265-281.

Galvão, K. N., M. J. B. F. Flaminio, S. B. Brittin, R. Sper, M. Fraga, L. Caixeta, A. Ricci, C. L. Guard, W. R. Butler, and R. O. Gilbert. 2010. Association between uterine disease and indicators of neutrophil and systemic energy status in lactating Holstein cows. J. Dairy Sci. 93:2926-2937.

Giuliodori, M. J., R. Magnasco, D. Becu-Villalobos, I. Lacau-Mengido, C. Risco, and R. de la Sota. 2013. Metritis in dairy cows: Risk factors and reproductive performance. J. Dairy Sci. 96:3621-3631.

Huzzey, J. M., T. Duffield, S. LeBlanc, D. Veira, D. Weary, and M. von Keyserlingk. 2009. Short communication: Haptoglobin as an early indicator of metritis. J. Dairy Sci. 92:621-625.

Leon, L. R. 2002. Invited review: Cytokine regulation of fever: Studies using gene knockout mice. J. Appl. Physiol. 92:2648-2655.

Liboreiro, D. N., K. S. Machado, P. R. Silva, M. M. Maturana, T. K. Nishimura, A. P. Brandao, M. I. Endres, and R. C. Chebel. 2015. Characterization of peripartum rumination and activity of cows diagnosed with metabolic and uterine diseases. J. Dairy Sci. 98:6812-6827.

Mackowiak, P. A., J. G. Bartlett, E. C. Borden, S. E. Goldblum, J. D. Hasday, R. S. Munford, S. A. Nasraway, P. D. Stolley, and T. E. Woodward. 1997. Concepts of fever: Recent advances and lingering dogma. Clin. Infect. Dis. 25:119-138.

Markusfeld, O. 1987. Periparturient traits in seven high dairy herds: Incidence rates, association with parity, and interrelationships among traits. J. Dairy Sci. 70:158-166.

Martinez, N., F. S. Lima, R. S. Bisinotto, L. F. Greco, E. S. Ribeiro, F. Maunsell, K. N. Galvão, C. A. Risco, and J. E. P. Santos. 2012. Evaluation of peripartal calcium status, energetic profile, and neutrophil function in dairy cows at low or high risk of developing uterine disease. J. Dairy Sci. 95:7158-7172.

Ospina, P. A., D. V. Nydam, T. Stokol, and T. R. Overton. 2010. Evaluation of nonesterified fatty acids and $\beta$-hydroxybutyrate in transition dairy cattle in the northeastern United States: Critical thresholds for prediction of clinical diseases. J. Dairy Sci. 93:546554 .

Risco, C., and P. Melendez Retamal. 2011. Dairy Production Medicine. Wiley-Blackwell, Oxford, UK.

Sheldon, I. M., J. Cronin, L. Goetze, G. Donofrio, and H.-J. Schuberth. 2009. Defining postpartum uterine disease and the mechanisms of infection and immunity in the female reproductive tract in cattle. Biol. Reprod. 81:1025-1032.

Sheldon, I. M., G. S. Lewis, S. LeBlanc, and R. O. Gilbert. 2006. Defining postpartum uterine disease in cattle. Theriogenology 65:1516-1530.

Soriani, N., E. Trevisi, and L. Calamari. 2012. Relationships between rumination time, metabolic conditions, and health status in dairy cows during the transition period. J. Anim. Sci. 90:4544-4554.

Stangaferro, M. L., R. Wijma, L. S. Caixeta, M. A. Al-Abri, and J. O. Giordano. 2016a. Use of rumination and activity monitoring for the identification of dairy cows with health disorders. Part I. Meta- 
bolic and digestive disorders. J. Dairy Sci. 99:7395-7410. http:// dx.doi.org/10.3168/jds.2016-10907.

Stangaferro, M. L., R. Wijma, L. S. Caixeta, M. A. Al-Abri, and J. O. Giordano. 2016b. Use of rumination and activity monitoring for the identification of dairy cows with health disorders. Part II Mastitis. J. Dairy Sci. 99:7411-7421. http://dx.doi.org/10.3168/ jds.2016-10908.

Steiner, A. A., A. I. Ivanov, J. Serrats, H. Hosokawa, A. N. Phayre, J. R. Robbins, J. L. Roberts, S. Kobayashi, K. Matsumura, P. E. Sawchenko, and A. A. Romanovsky. 2006. Cellular and molecular bases of the initiation of fever. PLoS Biol. 4:e284.

Titler, M., M.G. Maquivar, S. Bas, E. Gordon, P.J. Rajala-Schultz, K. McCullough, and G.M. Schuenemann. 2013. Effect of metritis on daily activity patterns in lactating Holstein dairy cows. J. Dairy Sci. 96(E-Suppl. 1):647. (Abstr.)
Toni, F., L. Vincenti, A. Ricci, and Y. Schukken. 2015. Postpartum uterine diseases and their impacts on conception and days open in dairy herds in Italy. Theriogenology 84:1206-1214.

Urton, G., M. Von Keyserlingk, and D. Weary. 2005. Feeding behavior identifies dairy cows at risk for metritis. J. Dairy Sci. 88:28432849.

Wittrock, J. M., K. Proudfoot, D. Weary, and M. Von Keyserlingk. 2011. Short communication: Metritis affects milk production and cull rate of Holstein multiparous and primiparous dairy cows differently. J. Dairy Sci. 94:2408-2412.

Zwald, N. R., K. A. Weigel, Y. M. Chang, R. D. Welper, and J. S. Clay. 2004. Genetic selection for health traits using producerrecorded data. I. Incidence rates, heritability estimates, and sire breeding values. J. Dairy Sci. 87:4287-4294. 